\title{
Recurrence or Relapse of COVID 19 Infection
}

\section{Gary R Skankey, MD* and Christopher Breeden, MD}

Internal Medicine/Infectious Diseases and Intensive Medicine, UHS Valley Health System, USA

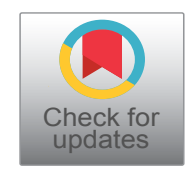

*Corresponding author: Gary R Skankey, MD, Internal Medicine/Infectious Diseases and Intensive Medicine, UHS Valley Health System, USA

\section{Case Presentation}

We present a patient who is a 67-year-old female who is morbidly obese (BMI 62), but otherwise healthy, who was admitted on 4/6/2020 (day 0 ) with a five day history of fever and diarrhea, followed by 24 hours worth of increasing shortness of breath, dry cough, nausea, and dry heaves. A chest $x$-ray showed bilateral infiltrates consistent with congestive heart failure, however B-naturetic peptide was only $284(\mathrm{NL}<125)$. Blood work was as follows: WBC - 3420, ABG - 7.43/34/64 on 6 liters $\mathrm{O}_{2}$, Quantiferon - negative, Coccidioides IgG and IgM both negative, and Blood cultures - negative.

In view of the ongoing coronavirus pandemic, and in view of fever, hypoxia, and bilateral infiltrates, she was presumptively started on hydroxychloroquine plus azithromycin. She was also started on ceftriaxone for empiric treatment of community acquired pneumonia. A COVID-19 PCR was obtained from the nasopharynx. Two days later it came back positive. Because of worsening oxygenation on day 1 , she was placed on a BiPAP mask. She was intubated on day 2 , because of worsening hypoxia and labored respirations. Blood work at this point was as follows: LDH - 591 (NL 120 - 250), CRP 16.20 ( NL < 0.3), Ferritin - 1216 (NL 8 - 252), D-dimer - 3.46 (NL < 0.59), and Procalcitonin - 0.16 ( $\mathrm{NL}<0.04)$.

Ceftriaxone was discontinued on day 3 in view of a negative sputum culture, negative blood cultures, and low procalcitonin. As there was clinical evidence of cytokine storm, an interleukin- 6 level was obtained on day 4 , and the patient was given tocilizumab. No noticeable response was detected as a result of the tocilizumab. Two weeks later, we received the interleukin 6 result, which was markedly elevated at 1491.3, with the upper limits of normal being 15.5. This confirmed the diagno- sis of cytokine storm, but also raised the question why there was not a more robust response to the tocilizumab. The QT interval was monitored while she was on the hydroxychloroquine. It remained within the normal range. She received five days of hydroxychloroquine plus azithromycin.

The patient remained ventilator dependent until day 30 . While on mechanical ventilation, she required as high as $100 \% \mathrm{FiO}_{2}$ and a PEEP as high as 14 . She also developed sustained hypercapnia. Chest X-ray showed persistence of bilateral infiltrates. Severity of hypoxia and hypercapnia peaked on day 15 , after which she started to show gradual improvement. COVID-19 PCR's repeated on day 22 and day 25 were both negative. After extubation, her respiratory status improved to the point that she was weaned down to $2 \mathrm{~L}$ nasal cannula oxygen by day 32 . On day 34 , she was noted to be ambulating in the hallways without oxygen and doing well, with no signs of visible dyspnea. Her chest $X$-ray on that day, although not completely clear of infiltrates, had shown significant improvement compared. Laboratory results had significantly improved as follows: Ferritin 689 (NL 8 - 252), CRP - 1.0 (NL < 0.3), LDH - 333 (NL 120 - 250), and D-dimer - 2.40 ( $\mathrm{NL}<0.59)$.

On day 37 , she developed progressively worsening dyspnea and hypoxia. She developed new low-grade fevers and a heart rate is high as $138 \mathrm{bpm}$. Repeat chest $x$-ray showed newly developed bilateral infiltrates. On day 38 she was placed on $100 \%$ BiPAP mask. Blood work results from days 37 and 38 were as follows: BNP - 665 (NL < 125), CRP - > 19 (NL < 0.3), Ferritin - 1746 (NL 8-252), D-dimer - 1.66 (NL < 0.59), LDH - 780 (NL 120-250), Procalcitonin - $0.36(\mathrm{NL}<0.04)$, and $A B G$ 7.19/70/77 on $90 \% \mathrm{FiO}_{2}$ and PEEP of 10.

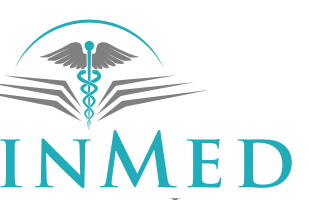

INTERNATIONAL LIBRARY

Citation: Skankey GR, Breeden C (2020) Recurrence or Relapse of COVID 19 Infection. J Infect Dis Epidemiol 6:138. doi.org/10.23937/2474-3658/1510138

Received: June 11, 2020: Accepted: July 01, 2020: Published: July 03, 2020

Copyright: (C) 2020 Skankey GR, et al. This is an open-access article distributed under the terms of the Creative Commons Attribution License, which permits unrestricted use, distribution, and reproduction in any medium, provided the original author and source are credited. 
Out of concern that she may have developed a hospital acquired bacterial pneumonia, she was started on meropenem plus vancomycin. Later on day 38 , she was once again intubated. Both blood in sputum cultures were repeated and came back no growth. A COVID-19 PCR was obtained from the nasopharynx and was positive. A serum COVID-19 antibody was also drawn and also resulted as positive. Now that remdesivir was available, she was started on day 40 , with a $200 \mathrm{mg}$ load followed by $100 \mathrm{mg}$ IV every 24 hours. She responded well to the remdesivir with improving oxygenation and $\mathrm{CO}_{2}$ levels. She remained intubated for only eight days, being extubated on day 46 . Final C-reactive protein was 1.7 and LDH was 347. Ferritin remained elevated at 1734. D dimer remains low throughout the admission due to prolonged heparin drip converted to oral Apixaban in the later weeks.

\section{Discussion}

SARS-CoV2 (CoVID 19) has been the cause of a worldwide pandemic. Reports have circulated of COVID-19 infected patients becoming PCR negative, and then becoming positive again. Concern was raised that these patients may be developing a second infection with the virus. However, in all of these cases, the re-emergence of the positive PCR was not accompanied by clinical symptoms. It was therefore surmised that the negative $P C R$ was a false negative, and that the later positive PCR was only detecting fragments of viral RNA remaining in the patients' secretions, without there being viable virus.

We here present a case of a patient who had severe clinical disease, recovered, had two negative COVID-19 PCR's, then relapsed with symptomology consistent with that of COVID-19 respiratory infection, as well as a repeat PCR that was positive for COVID-19. Evaluation of this case gives rise to three significant questions; 1 ) How sensitive and specific is the COVID-19 PCR? 2) Does a positive COVID-19 IgG indicate immunity to the virus? 3 ) Was this a true relapse of infection?

The COVID-19 nucleic acid amplification (NAA or PCR) employed in this case was run by LabCorp. The sensitivity of this test may be subject to collection error. In other words, if it is not collected appropriately, a false negative may result. This patient had two sequential PCR's that were negative, each obtained by a different nurse, reducing the likelihood of false negative. Although extremely sensitive and specific in early disease, in the convalescent patient, the COVID-19 PCR is subject to false positivity with regard to the presence of active, viable virus. A positive PCR weeks after resolution of symptoms is likely to be detecting remnants viral RNA residing in the respiratory secretions of patients in the absence of viable virions. A study done in Munich, Germany, and published in the journal, Nature, on 4/1/2020 [1] looked at patients with mild disease. Presence of subgenomic messenger RNA (sgRNA) was studied, which remain in COVID-19 infected cells and are not incorporated into the virions. The presence of sgRNA indicates active infection. The investigators discovered that the sgRNA disappeared after 5 to 11 days after onset of symptoms. This suggests that when the LabCorp COVID-19 PCR is positive, after the patient has been ill for at least one week, has an increasing likelihood of detecting residual fragments of RNA, and is not representative of continued infection. It is not known whether detection of sgRNA will be extended longer than 11 days in more severely infected patients. In our patient, could the repeat positive COVID-19 PCR have detected inactive viral particles, and therefore, this relapse is not an infection at all? Or, was this an actual relapse of the viral infection?

Antibodies to COVID-19 become detectable within 1 to 3 weeks after onset of symptoms [2]. As antibody levels increase, the viral load decreases. Interestingly, IgG and IgM rise at the same time rather than IgM levels increasing first, followed by IgG, as with other infections. Hence, the current essay only tests for IgG. A study of neutralizing antibodies during the 2003 SARS epidemic, revealed that they remained detectable on average for two years after infection [3]. The CDC emphasizes [2] that whether there is a correlation between seropositivity and immunity against repeat infections with COVID 19, is yet to be determined. Our patient had detectable IgG antibodies on the first day of the relapse of her infection. Her development of a clinical syndrome consistent with that of an active COVID-19 infection in the presence of anti-COVID-19 IgG is either explained by lack of a neutralizing antibody in response to the initial infection, which is not detected by the assay, or neutralizing antibodies were developed, and this relapse is not representative of an active viral infection, but a post-infectious immune mediated reaction. Additionally, the current assay is a qualitative and not a quantitative test. Could she have developed and inadequate response with a low titer of neutralizing antibody?

If the relapse episode was not due to an active infection, then the best explanation would be a post-infectious immune mediated response. Post-infectious immune mediated responses related to COVID-19 infections have been reported [4-6]. However, an immune mediated respiratory response, mimicking that of acute COVID-19 viral pneumonia, has not been reported. In our patient, There was acute development of fever, tachycardia, profound hypoxia, hypercapnia, bilateral infiltrates, marked increase in C-reactive protein, and marked increase of ferritin, which is what is encountered in acute COVID-19 infection. A minimal increase in the procalcitonin is a strike against a hospital acquired pneumonia. Additionally, blood in sputum cultures were no growth. The minimally elevated BNP makes pulmonary edema or congestive heart failure less likely. These findings provide strong evidence for acute infection. Her presentation was most consistent with a relapse of the 
viral infection, after it had been cleared from respiratory secretions, and in the presence of positive COVID-19 IgG, suggesting that the IgG in this assay probably does not represent a neutralizing antibody. More research needs to be undertaken to understand the pathophysiology of COVID-19 infection and development of immunity to the virus.

\section{References}

1. Wölfel R, Corman VM, Guggemos W, Seilmaier M, Zange S, et al. (2020) Virological assessment of hospitalised patients with COVID-2019. Nature 581: 465-469.
2. https://www.cdc.gov/coronavirus/2019-ncov/lab/resources/ antibody-tests-guidelines.html

3. https://www.ncbi.nlm.nih.gov/pmc/articles/PMC7184973/

4. https://labblog.uofmhealth.org/rounds/rare-covid-related-inflammatory-disease-affecting-children

5. https://www.medpagetoday.com/infectiousdisease/ covid19/86393

6. https://www.medpagetoday.com/infectiousdisease/ covid19/86029 